\title{
Evolution of a Unique Systems Engineering Capability
}

\section{INCOSE International Symposium}

\author{
Robert M. Caliva \\ James A. Murphy \\ Kyle B. Oswald
}

The INL is a

U.S. Department of Energy

National Laboratory

operated by

Battelle Energy Alliance

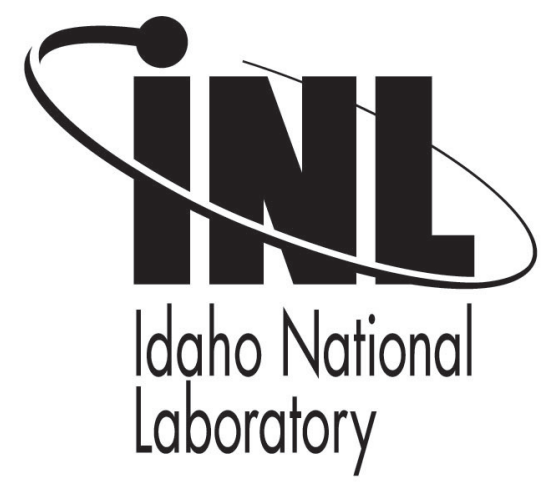

\section{June 2011}

This is a preprint of a paper intended for publication in a journal or proceedings. Since changes may be made before publication, this preprint should not be cited or reproduced without permission of the author. This document was prepared as an account of work sponsored by an agency of the United States Government. Neither the United States Government nor any agency thereof, or any of their employees, makes any warranty, expressed or implied, or assumes any legal liability or responsibility for any third party's use, or the results of such use, of any information, apparatus, product or process disclosed in this report, or represents that its use by such third party would not infringe privately owned rights. The views expressed in this paper are not necessarily those of the United States Government or the sponsoring agency. 


\title{
Evolution of a Unique Systems Engineering Capability
}

\author{
Robert M. Caliva \\ 208-526-4653 \\ Robert.Caliva@inl.gov
}

\author{
James A. Murphy \\ 208-526-4453 \\ James.Murphy@inl.gov
}

\author{
Kyle B. Oswald \\ 208-526-4863 \\ Kyle.Oswald@inl.gov \\ Idaho National Laboratory \\ 2525 N. Fremont Ave. \\ Idaho Falls, ID 83415-3780 \\ Copyright ( 2010 by Battelle Energy Alliance, LLC. Published and used by INCOSE with permission.
}

\begin{abstract}
The Idaho National Laboratory (INL) is a science-based, applied engineering laboratory dedicated to supporting U.S. Department of Energy missions in nuclear and energy research, science, and national security. The INL's Systems Engineering organization supports all of the various programs under this wide array of missions. As with any multifaceted organization, strategic planning is essential to establishing a consistent culture and a value discipline throughout all levels of the enterprise. While an organization can pursue operational excellence, product leadership or customer intimacy, it is extremely difficult to excel or achieve best-in-class at all three. In fact, trying to do so has resulted in the demise of a number of organizations given the very intricate balancing act that is necessary. The INL's Systems Engineering Department has chosen to focus on customer intimacy where the customer's needs are first and foremost and a more total solution is the goal. Frequently a total solution requires the employment of specialized tools to manage system complexity. However, it is only after understanding customer needs that tool selection and use would be pursued. This results in using both commercial-off-the-shelf (COTS) tools and, in some cases, requires internal development of specialized tools. This paper describes how a unique systems engineering capability, through the development of customized tools, evolved as a result of this customer-focused culture. It also addresses the need for a common information model or analysis framework and presents an overview of the tools developed to manage and display relationships between entities, support trade studies through the application of utility theory, and facilitate the development of a technology roadmap to manage system risk and uncertainty.
\end{abstract}

\section{Introduction and Background}

Introduction. The INL's Systems Engineering (SE) Department embarked on a comprehensive strategic planning process, resulting in the creation of a strategic map, which included the vision and mission shown in Figure 1. This set the strategic framework within which key roles and enablers could be defined. 


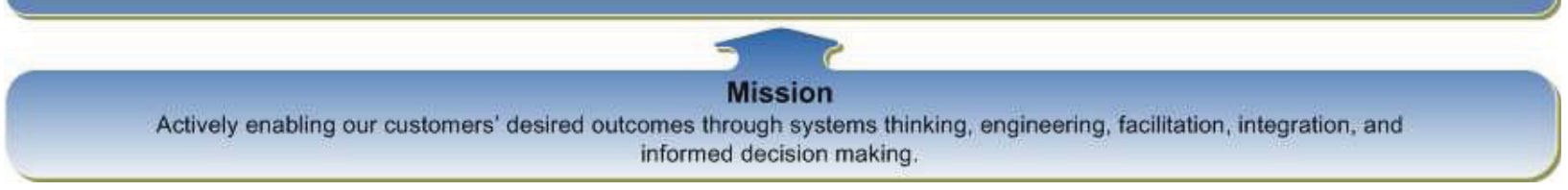

Figure 1. INL SE Department Vision and Mission

The department's charter is to provide systems engineering expertise to internal INL and Work for Others (WFO) program and project customers. Given this charter and the capabilities of the department, seven key roles were identified and are shown in Figure 2 that allow the organization to provide the quality SE support customers need and have come to expect. Customer intimacy became a priority where customer's needs were first and foremost. While the organization recognized a need to create and leverage processes and tools to achieve total solutions, tool development was not a key role or priority. However, it was recognized that informed decision making was an essential element to the customer's ability to achieve their desired outcomes and the use of tools became an important enabler. Box 2 in Figure 2 reflects that understanding.

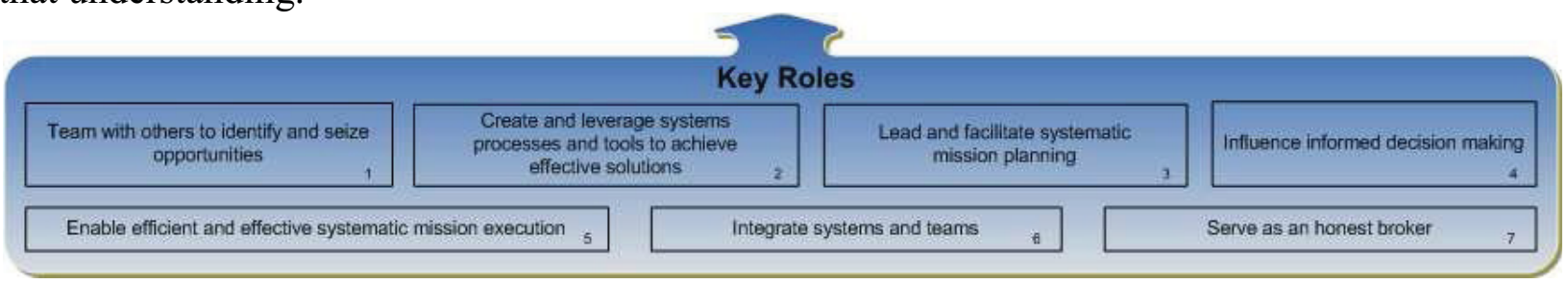

Figure 2. Key Department Roles

As reflected in Box 11 of Figure 3, a key Enabler to customer success was determined to be an integrated and connected view of project tools and methods. The key roles emphasized in the organizational strategy provide what the customer needs with strong and disciplined systems engineering resources. The enablers provide the department's resources the ability to meet and exceed customer's expectations.

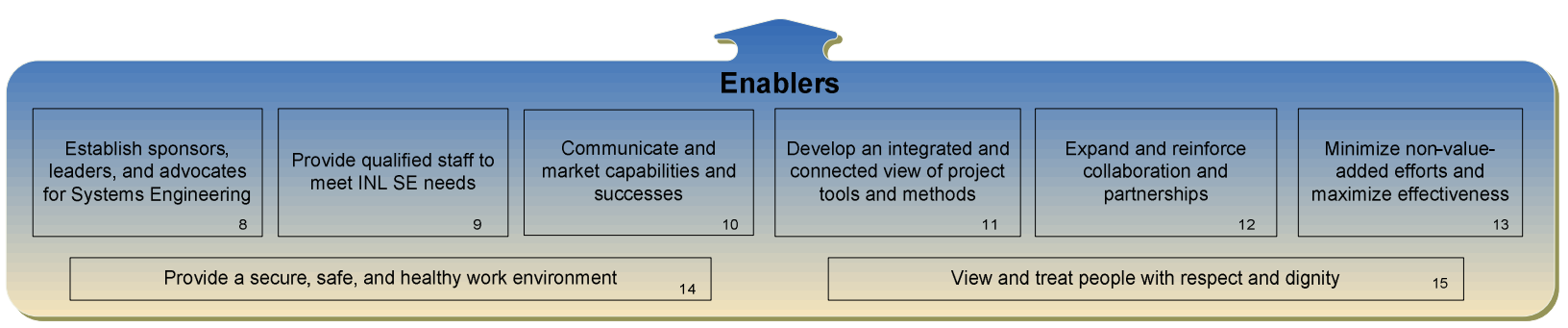

Figure 3. Enablers to Key Roles

Finally, under Learning and Growth, initiatives were identified to enhance the organization's ability to perform key roles as identified below in Figure 4, Box 20. This reflects a realization that a robust suite of tools was necessary to enable the customers' desired outcomes. 


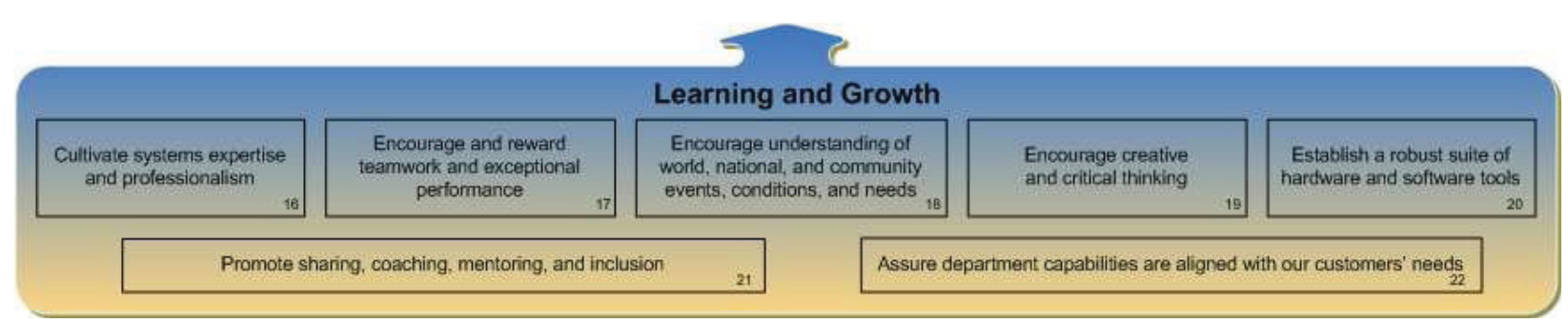

Figure 4. Learning and Growth

Background. Like many systems engineering organizations, communications with project managers can be challenging, especially in terms of marketing SE capabilities over the life-cycle of a project. Traditionally, project managers focus systems engineering on the beginning phases where requirements and functional analyses are required. In Figure 5, the SE Department has aligned the "V" diagram with the classical project phases and critical decisions (CD) over a typical project's life-cycle. Additionally, cross-cutting activities were identified, such as configuration management, modeling \& simulation, risk management and others, that often take place in support of more than a single SE function.

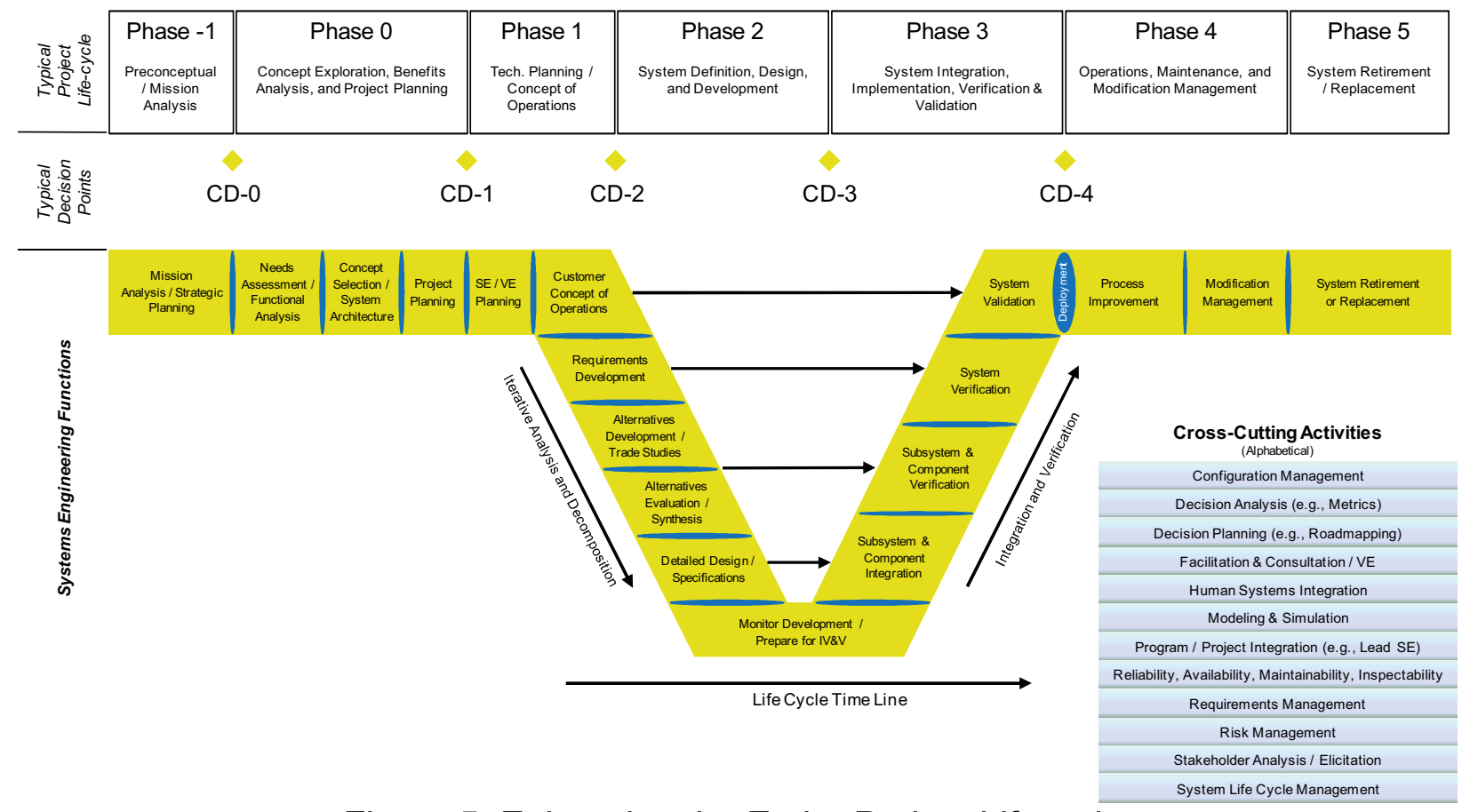

Figure 5. Enhancing the Entire Project Lifecycle

This has resulted in enhanced communications with project managers and a thorough understanding of underlying problems that could impact system development. As systems engineering support is provided across the "V" diagram and associated cross-cutting activities, the result is a more total solution to their needs. Although there are many COTS tools designed to help the system engineer perform these activities, customer needs often dictate that a more customized solution is required. While a project is moving through the early phases of the "V" diagram, mission needs, concept evaluation and selection, developing functions, performing 
trade studies, evaluating gaps in solution space, technology roadmapping, and risk management are areas where specialized tools were developed.

\section{Visual Tool Examples}

Zoned Analysis. In the 1960s, Dr. Milton E. Larson introduced a curriculum development model to "counteract the aimless evolvement of curriculum programs" in vocational technical education. Using the term Zoned Analysis, INL Systems Engineering has adapted Dr. Larson's model as a means of capturing and analyzing all aspects of the project to enhance the collaboration and planning of project activities. In practice, Zoned Analysis facilitates a gap analysis for the project and helps management quickly see the "big picture". Similarly, project personnel are better able to see their place and how they fit into the project whole. With this insight, functional requirements can be quickly identified and all deliverables linked back to the requirements. The results of Zoned Analysis become the source data for subsequent SE functions and tools. Figure 6 provides an example of a Zoned Analysis chart.

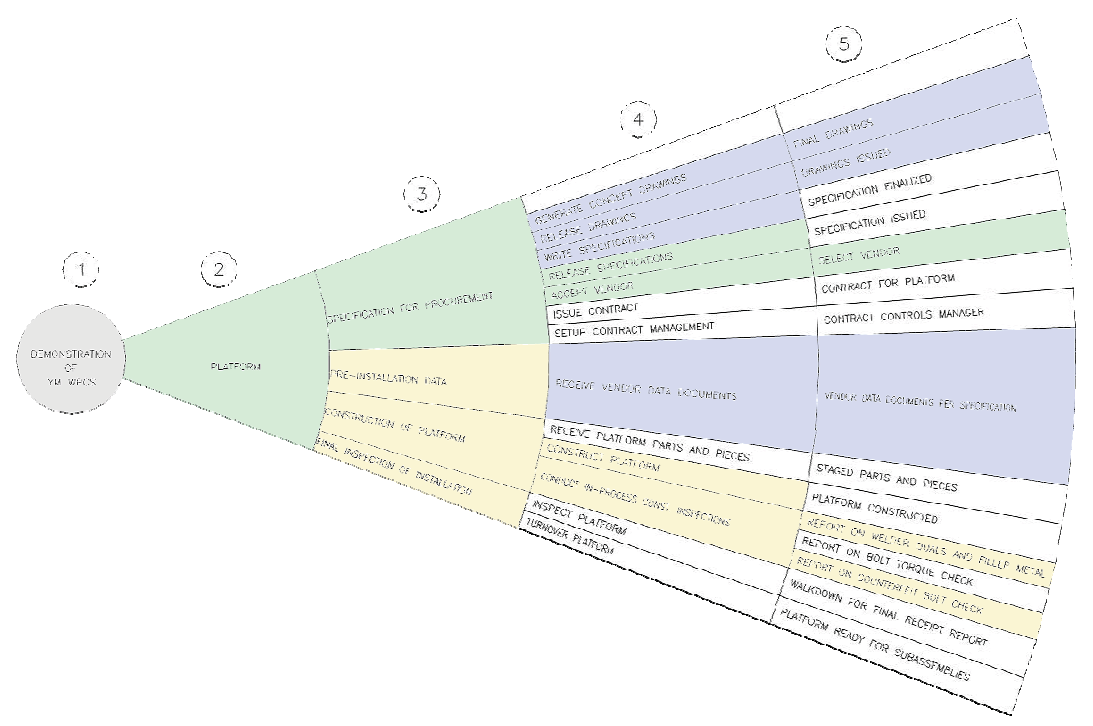

Figure 6. Example of a Zoned Analysis Chart

QuickCompare. The genesis of limited tool development within the SE Department was a tool called QuickCompare, which was developed in-house by INL Systems Engineers. QuickCompare is a flexible tool that can be applied to a wide range of decision making situations. It helps document the decision making processes (e.g., define the problem, goals, alternatives, criteria, and weights; score alternatives; analyze results) and makes the decision analysis process faster, easier, and more transparent. QuickCompare provides the ability to evaluate alternatives from strictly qualitative to highly quantitative and contains the capability for performing sensitivity analysis and testing the validity of multiple "what if" scenarios. It also provides multiple comparative graphical outputs to facilitate better understanding of the impact of those scenarios on the decision to be made. An example of a QuickCompare visual table is at Figure 7. 


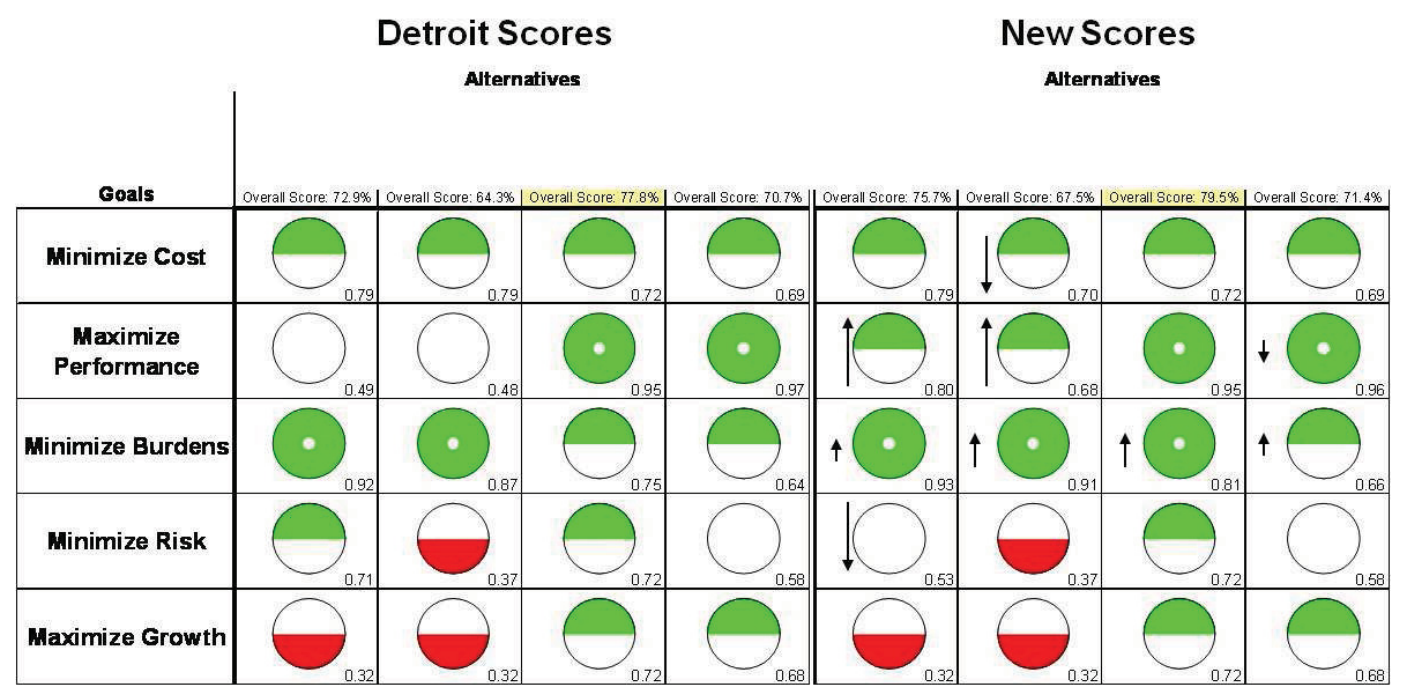

Figure 7. Example of a QuickCompare Table

\section{Traceable Gap Prioritization Analysis}

In 2006, a U.S. Army Current Force (CF) Fleet Management and Modernization (FMM) Initiative was launched to focus on technology insertion with the purpose of maximizing brigade and platform effectiveness specific to future force (FF) capability needs. The goal of the initiative was to support development of modernization plans, which serve as input to the Program Objective Memorandum (POM) cycle to modernize the CF Brigade Combat Teams (BCTs) with requisite capabilities that enable them to complete specific missions. Toward this end, it was recognized that impact on BCT-level system of systems (SoS) performance would be an important criterion in deciding which solutions to include in the modernization plans. The INL, a member of the Future Force Integrated Support Team (FIST) ${ }^{1}$ already conducting other Army analyses, was engaged to support the qualitative assessment of BCT baseline and enhanced performance. The INL support provided the impetus for developing a qualitative capability gap assessment approach and tool appropriate for use at an enterprise or SoS level.

Tool Purpose \& Overview. A relational database application was developed to support the PEO-GCS process discussed above and was named the Gap Relationship and Integration Planning (GRIP) Tool. It was programmed in Visual Basic for Applications (VBA) which provides a graphical user interface for working with the Microsoft Access ${ }^{\circledR}$ database as well as the necessary data capture and management for conducting the utility theory-based assessment. GRIP was designed for use by INL FIST team members to facilitate the data capture from Subject Matter Experts (SME) during workshop sessions and to provide rapid feedback on results—often within the same session.

The original data schema (see Figure 8) on which the tool is based was developed in Rational Requisite Pro $^{\circledR}$, which was used initially to manage the many data element types and their interrelationships. At present, GRIP effectively manages relationships between multiple data

\footnotetext{
${ }^{1}$ FIST is a consortium of Department of Energy laboratories including INL, Oak Ridge National Laboratory, and Sandia National Laboratories originally formed to provide systems engineering integration and SoS analysis support to the U.S. Army's Future Combat Systems (FCS) Program.
} 


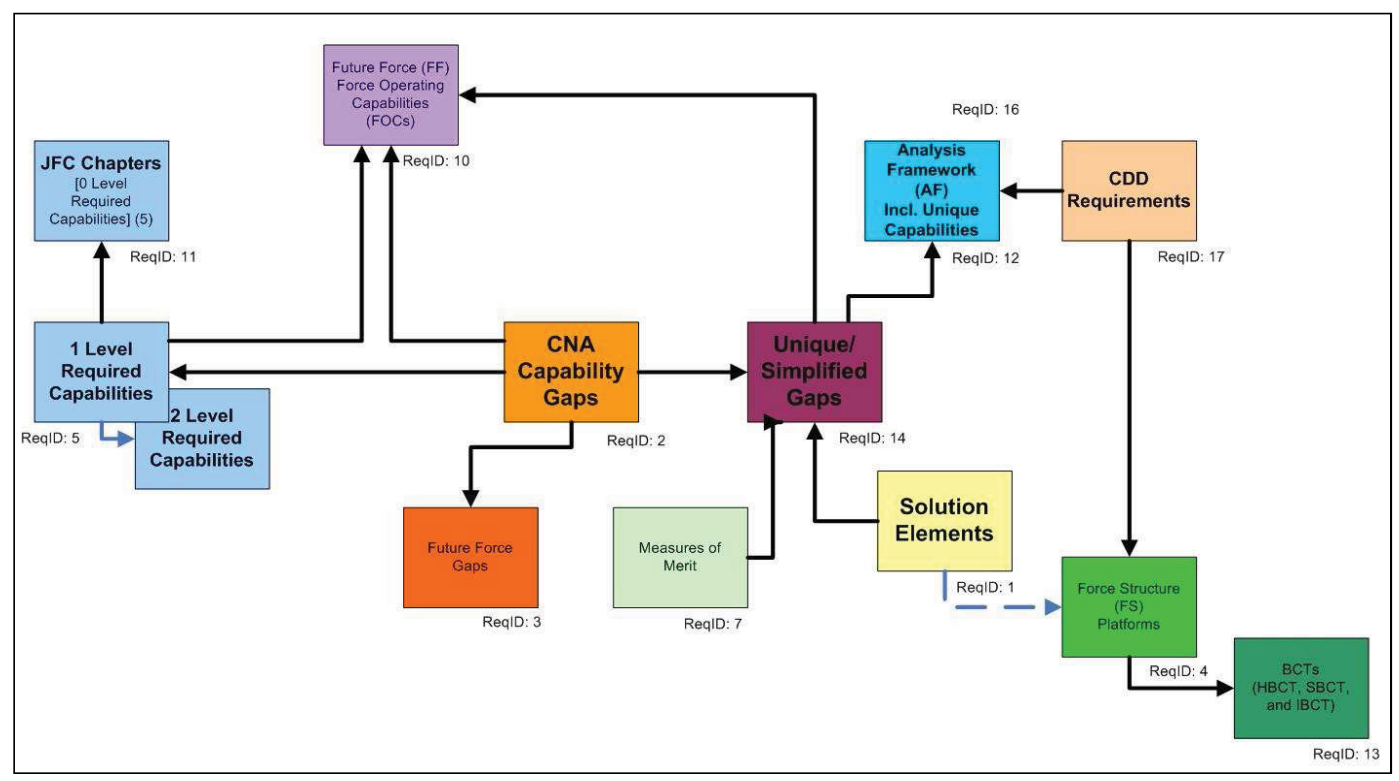

Figure 8. Partial GRIP Data Relationship Schema

element types including: required capabilities (RCs), RC gap statements, future force gaps, unique capabilities (i.e., the independent and non-redundant functional areas distilled from RC gap statements), unique/simplified gaps (i.e., the reconstituted functional and performance attribute deficiencies for the unique capabilities), BCT (enterprise) elements, and potential technology solutions for gap closure. The tool has the ability to display these mapped relationships (using a hierarchical tree structure) including data traceability back to multiple Army source documents. The tool also includes the ability for the user to look up or down the tree from a selected node of interest.

GRIP automates all of the mathematical calculations associated with performing the SoS capability gap qualitative assessment including normalization of importance weights, effectiveness score conversion to utility values, gap size calculations, and the scoring results rollup. It also provides the ability to store multiple assessments; each in the context of an associated mission.

GRIP also provides built-in functionality for charting and reporting results:

- Has multiple output charts for displaying results including baseline capability, initial capability gaps, capability organizational responsibility/ownership, enhanced capability performance assuming solution element implementation, solution element comparative effectiveness, and residual gaps.

- Displays assessment rollup results for the various levels within the analysis framework using Consumer Reports ${ }^{\circledR}$-style icons for easy comparison and identification of problem areas (shared from QuickCompare previously discussed).

Using the schema, information was collected and related to provide a full transparency of the gaps with the other elements that helped describe the actual problem. The focal point of the data gathering was to provide the ability to score the baseline. A capability performance level as well 
as an importance value was collected for two major missions. A weighted utility score was calculated for each of the gaps and then ranked from highest to lowest. When ranked this way, GRIP can produce a "paredo-type" chart as shown in Figure 9, identifying a knee in the curve after which there is less value or Return-On-Investment (ROI) when closing the gaps.

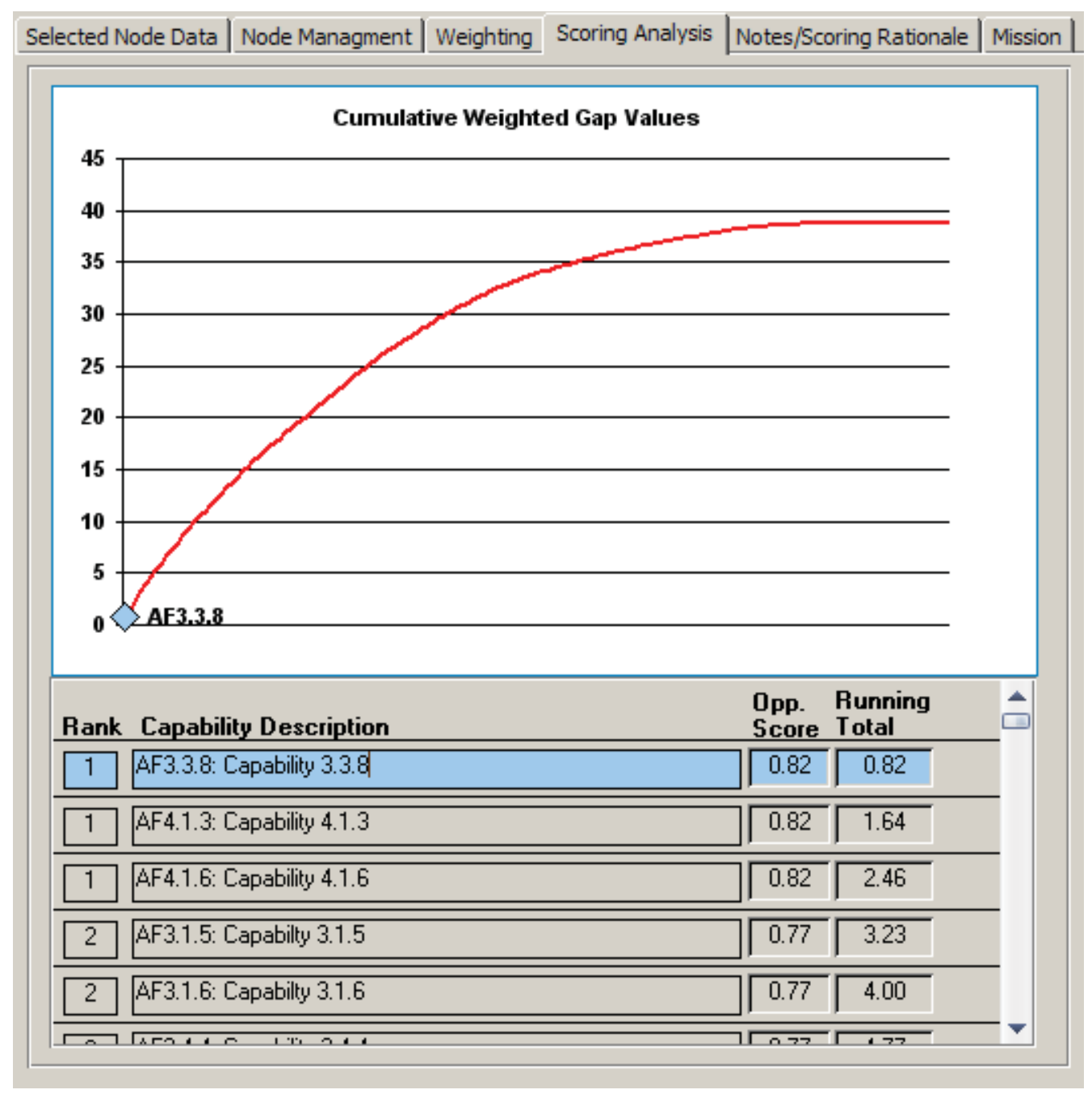

Figure 9. Cumulative Weighted Gap Values

Again, using SME and professional judgment, solutions can be evaluated using the same measure of effectiveness (MOE) performance criteria used to define the baseline deficiency. The new expected performance level creates a "delta" impact score (i.e., from the baseline score) for each alternative solution against the baseline gaps. This linkage provides the opportunity to capture where solutions have multiple affects on closing more than one gap and how much of the gap would be closed by one solution or another. Figure 10 provides an example of the CF FMM Initiative capturing solutions towards the AF 1.1.8 Capability where four materiel solutions were identified. In this example, a relative improvement was awarded toward closing this gap. A customized utility scale was used for the Army to award both the value to the baseline and alternative solutions toward future, often not fully defined requirements. Both the baseline and alternatives prioritization based on the methodology employed by GRIP was continuously 
validated by examining the output results with multiple critical and knowledgeable Army SMEs.

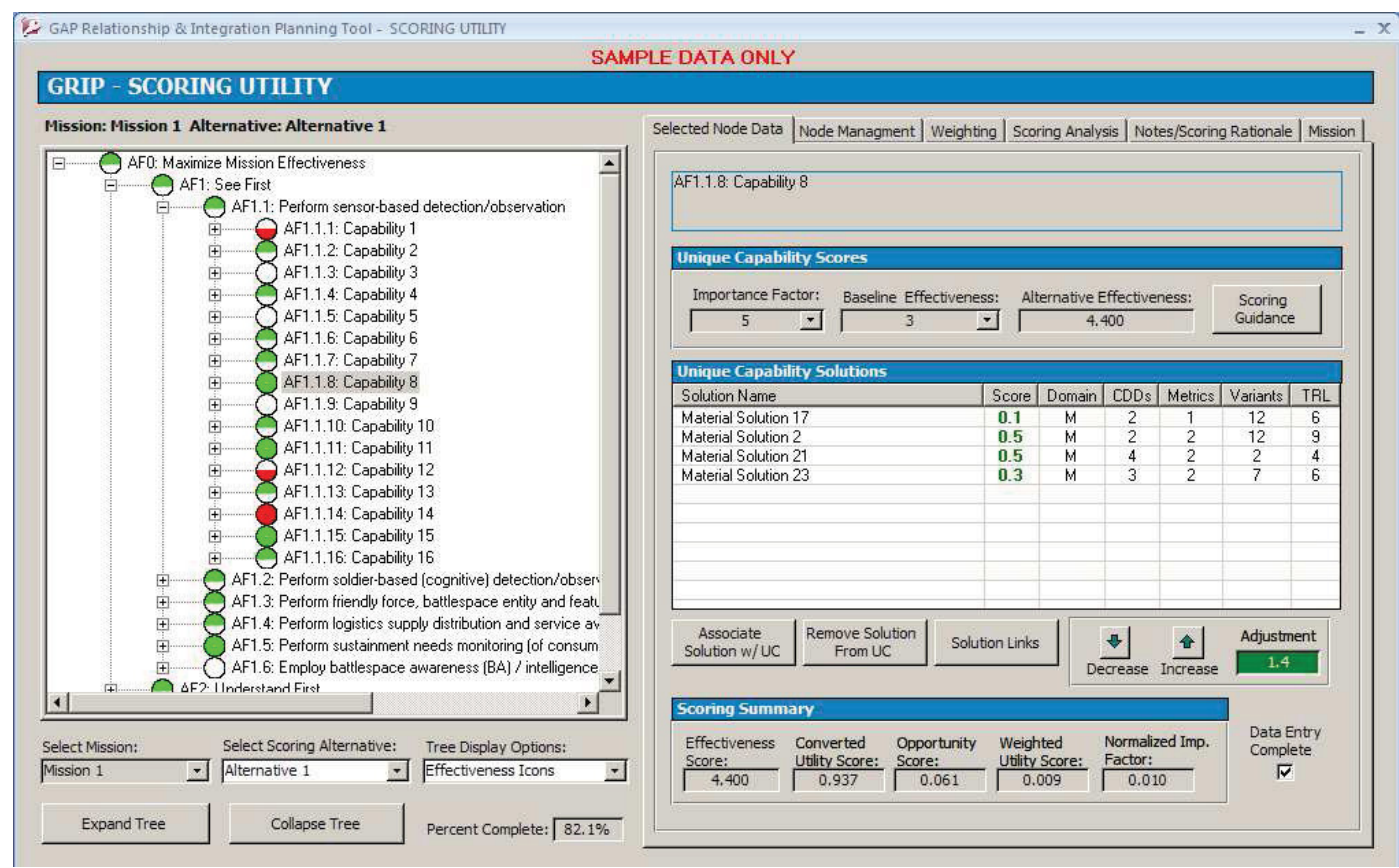

Figure 10. Solution Element Association and Scoring of Impact on Capability

However, as solution priorities were examined based on the largest value, a disconnect was discovered that required an additional function in GRIP. Several solutions that were scored were found to have low value scores due to the capability not being increased; rather, the solution provided a critical enabler to a high value solution. A prerequisite function was added so a solution that required enablers would ultimately promote the enabler just in front of when the solution would show up in the priority order. Figure 11 shows the results of the highest value alternatives in order from left to right with critical enablers promoted to the left of the solution with the greatest value. As budgeting is considered, this keeps the solutions logically tied and transparently displays the value of the solutions.

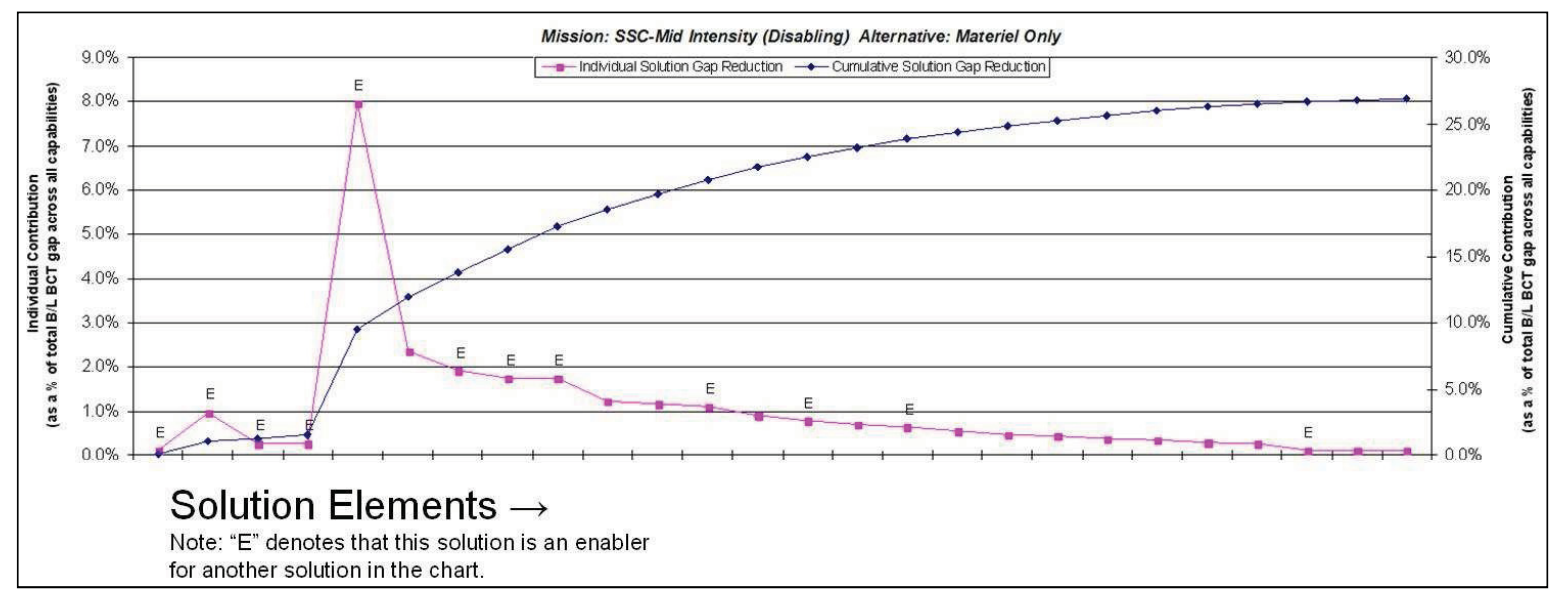

Figure 11. Solution Ranking Accounting for Precedence Relationships 
Based on the functionality of GRIP, its ability to cross-correlate a huge amount of critical data and relationships, and its impact on supporting informed decision making, several tailored applications have been developed to meet the specific needs of other projects. The GRIP functionality was expanded from gap priorities and prioritization of solutions to also provide project management functions and validation of performance at the system level.

The Strategic Milestones and Relationships Tracking (SMART) tool was developed for the U.S. Army Hit Avoidance System program to manage the complex relationships of program artifacts, evidence, reviews, tasks, status, and relationships to other project elements.

The Performance Rollup Tool (PRT) captures and evaluates a number of variables affecting a system's performance across multiple scenarios. System issues can be evaluated and prioritized for highest value to overall performance across each scenario.

The INL Portfolio Integration and Prioritization (iPIP) tool provides an ability to easily capture and analyze the interrelationship of organizational elements (called capabilities) that play a role in the overall strategy and tactics of the enterprise. The decision model portion of the iPIP manages the relationship between the programmatic users of the enterprise architecture, the organizations developing and maintaining the enterprise architecture, and the investment decisions made to maximize mission success and minimize programmatic risk to the enterprise.

\section{Roadmapping and Risk Management}

Roadmapping. Systems engineers at the INL have developed a specialized planning and technology roadmapping capability that provides the rigor and understanding needed for decision-makers to focus on critical uncertainties and make informed decisions. In short, the advanced roadmapping process provides the means to:

- Measure the relative merit of technologies

- Identify the key selection discriminators for down selecting technologies and designs

- Establish the long-term vision for maturing technologies toward deployment and operations

- Identify key uncertainties that reduce the ability to decide on technology with high confidence and ensure that the risk to the decisions are reduced sufficiently on the needed time cycle

- Identify risks early in the process and outline the tasks needed to resolve technical risks

- Accelerate the application of new technologies

- Minimize project costs and schedules

- Provide a defensible argument for acquisition choices.

These capabilities combine to form a technical risk reduction strategy, sometimes referred to as a Focused Roadmap (see Figure 12). The tools can be applied to technical or programmatic risk, including economic, stakeholder, and political risk. It was found that some development activities provided significant risk reduction, some provided multiple risk reductions, and some provides less reduction. 


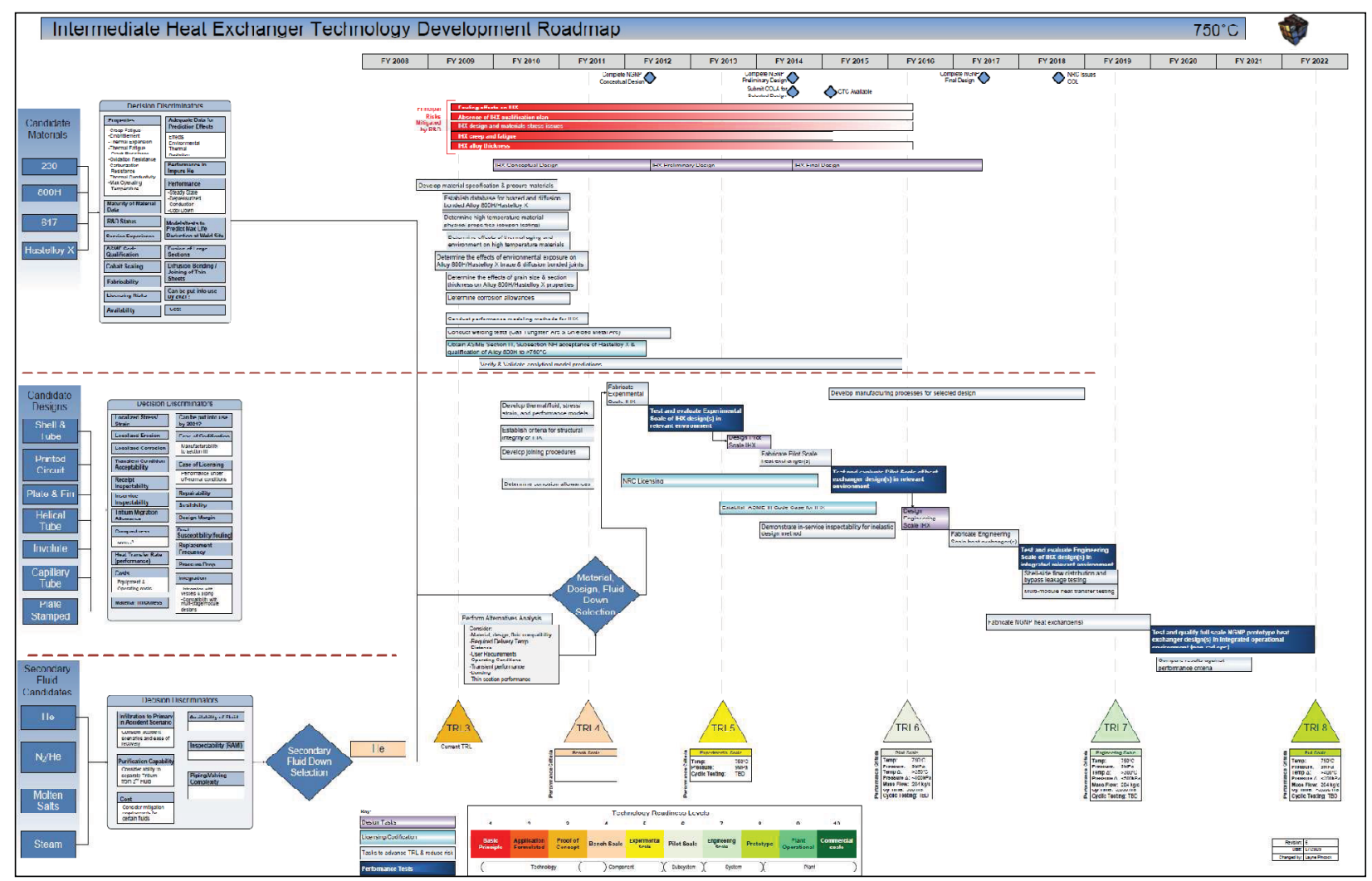

Figure 12. Focused Technology Development Roadmap for the NGNP IHX

Risk Management. To facilitate development and execution of risk management plans, an analytical software tool was developed leveraging the GRIP tool called the Risk Management System (RMS). The RMS provides conventional database utility including data maintenance, archiving, configuration control and query-ability. Additionally, the tool's design provides a number of unique functions and reporting capabilities that facilitate the development and execution of activities outlined in the risk management plan. Specifically, the RMS provides the capability to:

- Establish the risk baseline

- Document and analyze the risk reduction plan

- Track the current risk reduction status

- Organize risks by a reference configuration

- Inform decision making such as prioritizing risk reduction activities by those that best help the program reduce the largest critical uncertainties

It also provides a testing basis for the uncertainty reduction/risk reduction by defining the expected outcome of the risk reduction that can be compared to the actual results. 


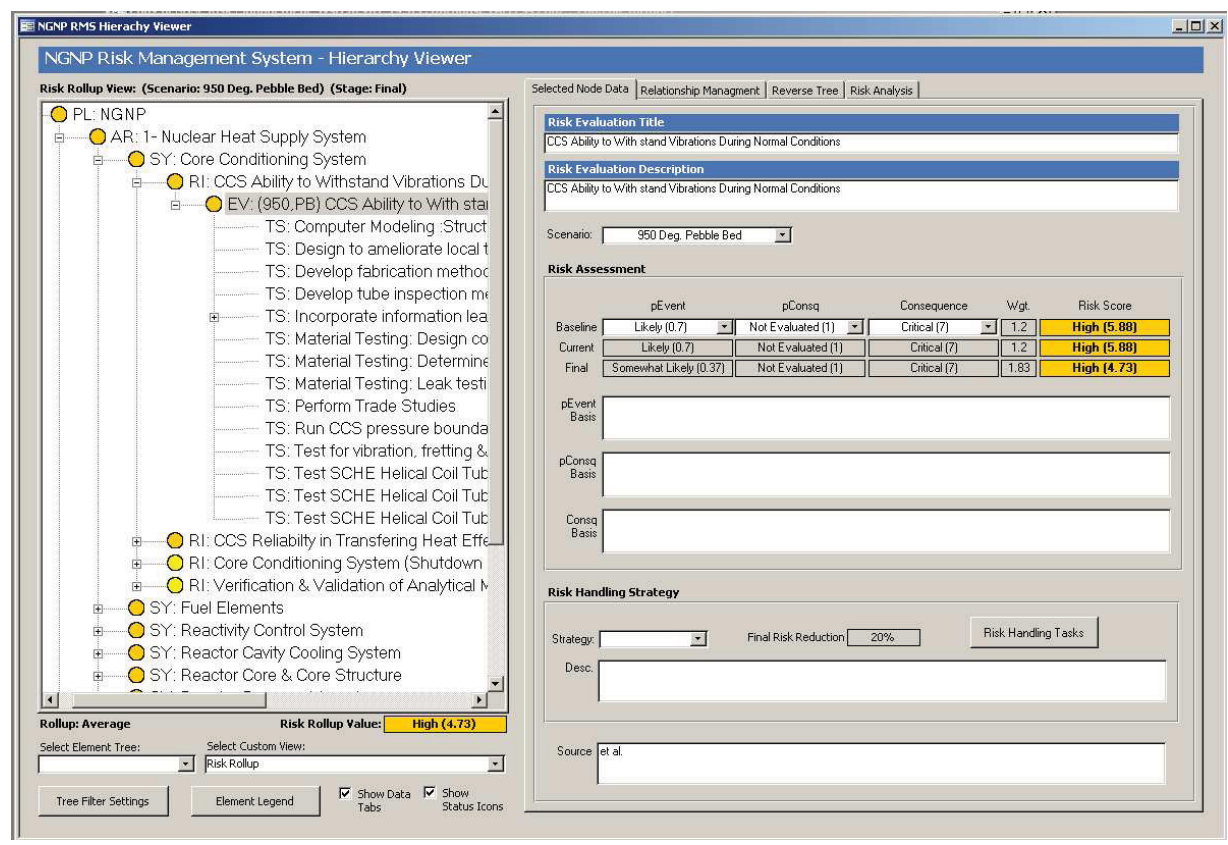

Figure 13. RMS Hierarchy Viewer

The RMS Hierarchy Viewer shown in Figure 13 provides a rollup/drilldown analysis capability that summarizes quantitative risk scores at various levels of granularity. The risk scores can be displayed for either the baseline, current status or the final projected risk. Risks can be rolled up by average or worse case for a selected design configuration. The tool's hierarchy tree also allows the visualization and analyses of the complex relationships between various data entities (e.g. tasks, team member assignments, and organization/category elements).

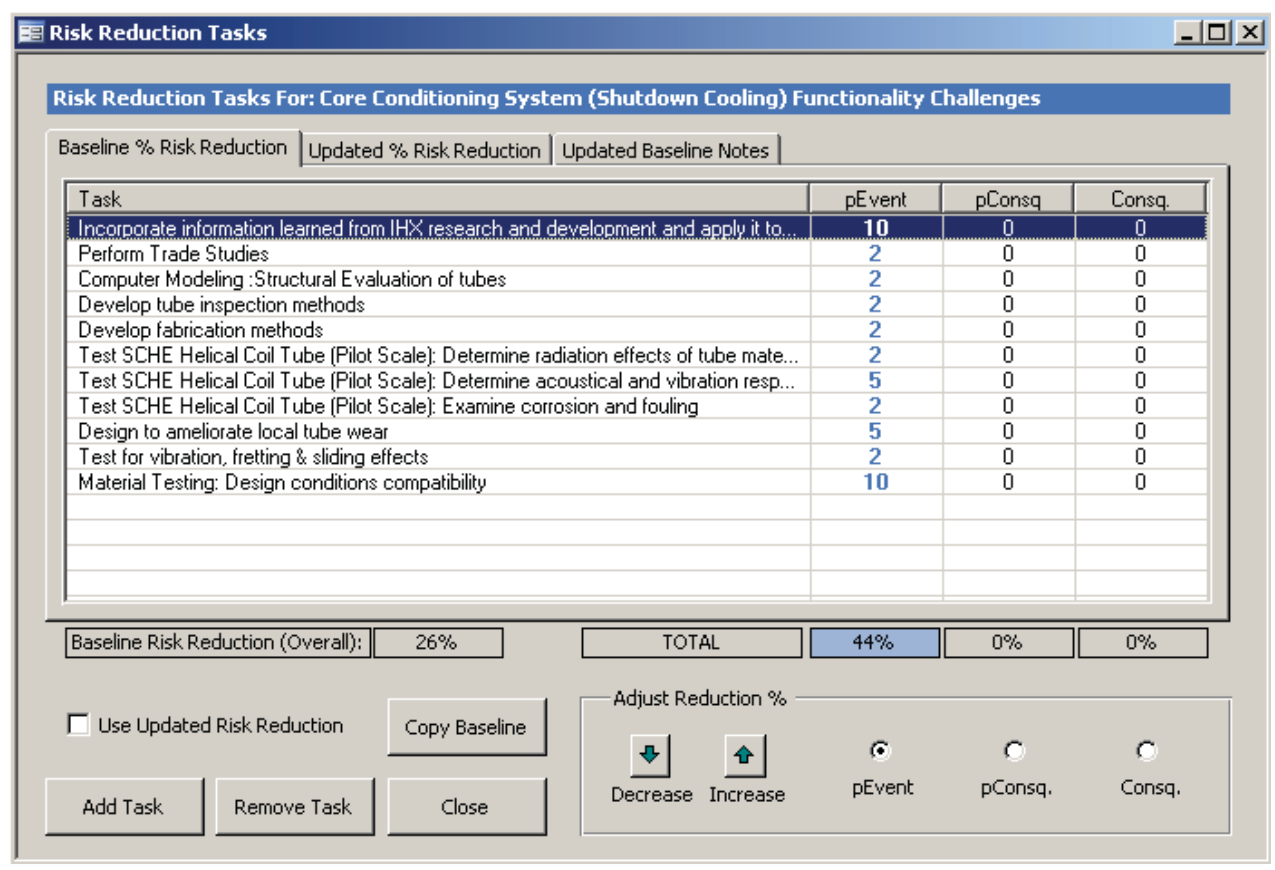

Figure 14. Risk Reduction Tasks 
The RMS provides the capability to outline and status a risk handling strategy for each identified risk. Risk reduction tasks are assigned to each risk item and the magnitude of risk reduction estimated for each associated task can be specified as shown in Figure 14. Status of the risk handling strategy is primarily based on the percent completion of risk reduction tasks. The status

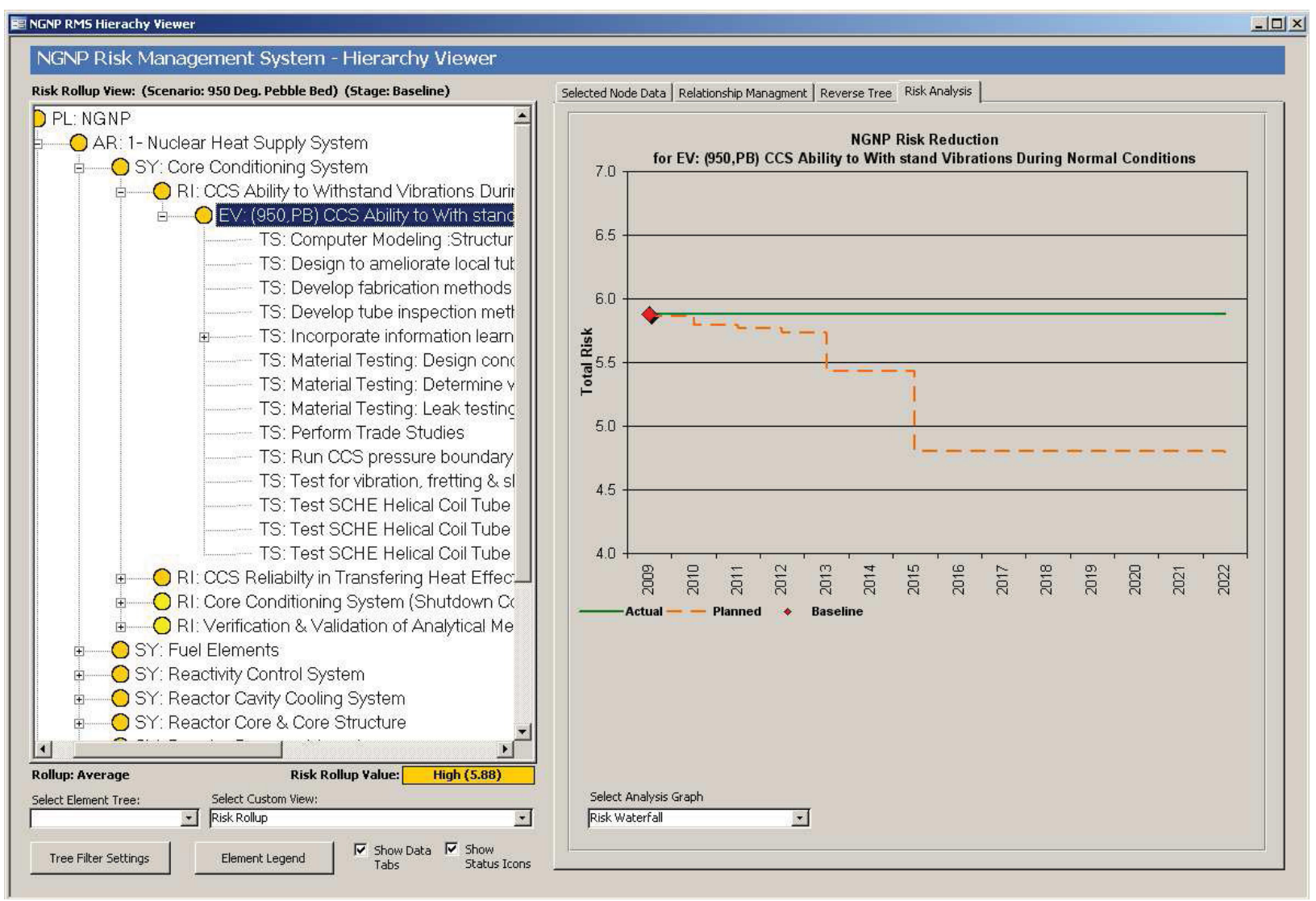

Figure 15. Risk Waterfall

of the strategy can also be seen graphically in a Risk Waterfall chart that displays the actual/current risk reduction versus the planned risk reduction over time (see Figure 15). For tasks that provide a reduction in risk for more than one risk item, the tool provides the ability to summarize its contribution across the entire risk plan. This capability makes it possible to rank order tasks by the magnitude of risk reduction provided for the entire project. This rank ordering of tasks then provides input into project planning and prioritization.

Additional RMS functionality includes the ability to analyze and track relational mapping between project risks and technology development activities and facilitating gap identification in planning R\&D activities.

\section{Alignment with the "V" Diagram}

The tools developed at the INL, as well as the COTS tools that are used, overlay nicely onto the SE "V" diagram in Figure 16 to communicate to the SE Department and customers what is available and how they could be useful performing SE functions. 


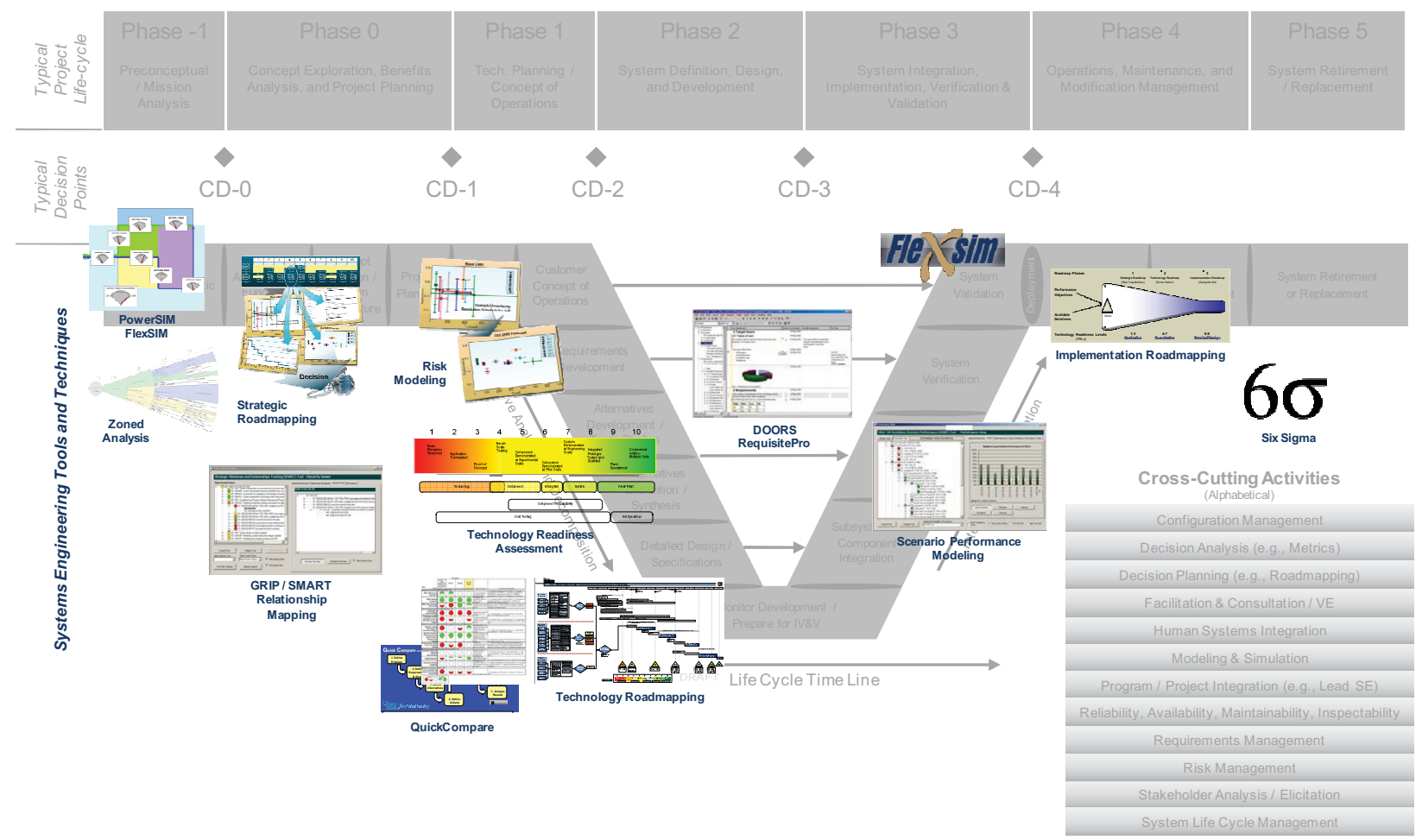

Figure 16. Tools and their Application within a Project Life-Cycle

\section{Lessons Learned}

The following lessons learned were documented in relation to the use of the tools described in this paper.

Required Support. The GRIP tool was very effective at handling a large number of capabilities and the associated data relationships. However, with a large number of capabilities, two missions and multiple solution elements to assess, the breadth and depth often required SMEs to be sequestered to understand and validate the assessment. Secondly, somewhat because GRIP and iPIP contained data from so many SMEs, no one organization or individual understood the net benefit of using an integrated system on a continuous basis. This was partially due to the organizations already having decision processes that often were contrary in conclusion to the output of the enterprise-level database due to local optimization. There should be considerable planning and communication with the decision processes in place as to how to integrate higher level, cross-integrating views and analyses.

Information Updates. Managing the changes to supporting or source data over time for purposes of maintaining traceability proved to be a challenge partially due to the organizational entropy with their existing processes. The potential for this situation to occur should be considered during the planning phase when there is ample time to incorporate mechanisms to facilitate data updates by getting organizations to use the information from the tools.

Prototyping. Many of the analytical methods applied to derive comprehensive customer solutions required evolutionary tailoring as the domain of the challenge became better understood. As such, the design of tools used to support these solutions must also evolve. This 
required a rapid prototyping approach to software design and implementation where the software tool was continually refined and rebuilt. Rapid prototyping provided several advantages including: immediate customer involvement/engagement which allowed for direct feedback on methodology approaches and specifications, less chance of miscommunication as complicated data and analysis were more instantly visualized, and of course a more timely delivery of the final product.

Teaming. The qualitative process, which relies on SME input, is enabled when needed participants are identified and become part of the analysis team. Otherwise, the lack of an established team with roles and responsibilities will create competing priorities for the SMEs. To formalize SME support when working with organizations like the U.S. Army, a method such as a formal task order, or some other command level of commitment is critical so requests for information can be satisfied within the timeframe needed.

Workshops. Workshops with SMEs are an effective way of not only capturing essential input, but facilitate communication across the enterprise both during the workshop as a result of the discussion and afterward due to the information that was uncovered. Due to the broad base of knowledge needed for an enterprise assessment like what was done for the Army, capability importance and effectiveness values need to be first obtained from individuals outside a team session. With this strawman data, the workshops become critical for normalizing that input and for promoting acceptance of the assessment results. These workshops should be planned in detail, aggressive and facilitated to maximize the limited time most SMEs can afford to provide.

Defining the Mission. The mission of the system must be well defined and documented such that it can ultimately be used to describe the environment, circumstances, and value space wherein the capabilities will be employed. A well defined mission will facilitate the decomposition of necessary functions that is needed for compiling the list of capabilities to be assessed.

\section{Conclusion}

Across any large organization, strategic planning is essential to establish a consistent culture throughout all levels of the enterprise. While an organization can pursue operational excellence, product leadership or customer intimacy, it is extremely difficult to excel or achieve best-in-class at all three. The INL's Systems Engineering Department is focused on customer intimacy where the customer's needs are first and foremost and a more total solution is the goal. Only after understanding what the customer really needed would tool selection and use be pursued. Ultimately, a unique systems engineering capability, through the development of customized tools, evolved as a result of this customer-focused culture. Clearly defining the customer's problem and desired end state, ensuring interfaces were well defined, traceability established, metrics identified and utility theory incorporated where needed with specific and unique graphical products resulted in frequent and engaged communications with the customer. Diligence is necessary to preclude an over reliance on these tools and a shift from a customer intimate organization to product leadership or a tool development enterprise. After the success of Zoned Analysis, QuickCompare, and the GRIP tool on the CFFMM Initiative documenting a process and facilitating navigation of the data and information, other tools were spun off in a similar manner to better enable INL's customers to make informed and defensible decisions. 


\section{Future Applications or Application to Other Domains}

In support of the Department of Homeland Security, a prototype system security evaluation tool was built on an EXCEL platform and provides the user the ability to quickly explore alternatives to proactively improve system security or reactively consider alternatives against an event. Future development will assist the customer in evaluating alternatives quickly across multiple objectives of a decision framework.

\section{Acknowledgements}

This manuscript has been authored by Battelle Energy Alliance (BEA), LLC under Contract No. DE-AC07-05ID14517 with the U.S. Department of Energy. The publisher, by accepting the article for publication, acknowledges that the United States Government retains a nonexclusive, paid-up, irrevocable, world-wide license to publish or reproduce the published form of this manuscript, or allow others to do so, for United States Government purposes.

\section{References}

Capability Needs Assessment (CNA) Database. U.S. Army Capabilities Integration Center (ARCIC). U.S. Army Training and Doctrine Command (TRADOC).

Enterprise. 1993. American Heritage College Dictionary. $3^{\text {rd }}$ Edition. New York: Houghton Mifflin Company.

Treacy, M. and Wiersema, F., 1995. Discipline of Market Leaders. Reading, MA: Perseus Books.

\section{Biographies}

Robert M. Caliva is a project manager in the BEA Systems Engineering Department at the INL. $\mathrm{He}$ has a BS in General Engineering from the United States Military Academy, an MBA from the University of Central Florida and an MS in Systems Engineering from the University of Idaho.

James A. Murphy is a senior systems engineer in the BEA Systems Engineering Department at the INL. He has a BS in Chemistry and Biology from the College of Idaho and an MS in Chemistry from the University of Idaho. James has 29 years of experience supporting DOE and DOD system optimization and problem solving to include technology development, infrastructure investment and portfolio analysis, programmatic, technical, and environmental risk management, threat defeat analysis, and facilitation of disciplined decision making.

Kyle B. Oswald is a senior systems engineer in the BEA Systems Engineering Department at the INL. He has a BS in Mechanical Engineering from Brigham Young University and an MS in Mechanical Engineering from the University of Idaho. 